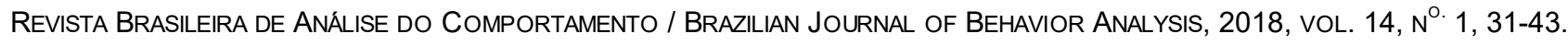

\title{
TRANSFERÊNCIA DE FUNÇÃO E REORGANIZAÇÃO DE CLASSES DE EQUIVALÊNCIA RELACIONADAS A GÊNERO E PROFISSÕES
}

\section{TRANSFER OF FUNCTIONS AND REORGANIZATION OF EQUIVALENCE CLASSES RELATED TO GENDER AND PROFESSIONS}

\author{
AlESSANDRA PINTO ROSENDO - ORCID 0000-0002-0894-9697
}

Raquel MaRIA de Melo - ORCID 0000-0003-2414-0633

\author{
UNIVERSIDADE DE BRASÍLIA E

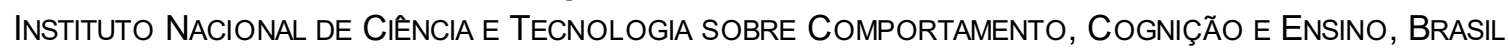

\section{RESUMO}

Foi investigado o efeito de treinos de reversão na reorganização de classes com estímulos abstratos, adjetivos e profissões relacionadas aos gêneros masculino e feminino. Na Etapa 1, os participantes dos grupos Experimental 1 (N=8) e Experimental $2(\mathrm{~N}=8)$ foram expostos a treinos e testes de formação de classes com o procedimento de pareamento ao modelo com atraso. Para o Grupo Experimental 1, na Etapa 2 foram realizados treinos de reversão e testes de reorganização de classes. O Grupo Controle $(\mathrm{N}=32)$ não foi exposto a nenhum dos treinos e testes. Os três grupos avaliaram os estímulos dos conjuntos A (profissões) e D (símbolos abstratos) com o Diferencial Semântico. Na Etapa 1, foi verificada a formação de classes de equivalência para os dois grupos experimentais. Na Etapa 2, os participantes do Grupo Experimental 1 atingiram o critério de reorganização de classes (91\% de acertos) apenas para as relações de simetria e nos demais testes os escores foram próximos (83\%) para seis participantes. Quando comparado com o Grupo Controle, as avaliações no Diferencial Semântico dos estímulos A e D foram parcialmente coerentes com as classes de equivalência (Grupo Experimental 2) e com os treinos de reversão e testes de reorganização de classes (Grupo Experimental 1), com transferência de função para algumas escalas. Os resultados mostraram a generalidade dos procedimentos para investigar classes de estímulos relacionadas a profissões e gêneros, porém controles metodológicos devem ser aprimorados em relação a verificação da história pré-experimental com os estímulos utilizados e a quantidade de relações revertidas.

Palavras-chave: equivalência de estímulos, reorganização de classes, transferência de função, pareamento ao modelo com atraso, diferencial semântico.

\section{ABSTRACT}

This study investigated the effects of reversal training on the reorganization of classes with abstract stimuli, adjectives and professions related to masculine, feminine or both genders. In Stage 1, participants of Experimental 1 $(\mathrm{N}=8)$ and Experimental $2(\mathrm{~N}=8)$ groups were exposed to training and tests of class formation with a delayed matching to sample (DMTS) procedure. For Experimental 1 group, in Stage 2, reversal training and tests of class reorganization were conducted. The Control group $(\mathrm{N}=32)$ was not exposed to training and tests. The three groups evaluated stimuli A (professions) and D (abstract symbols) with the Semantic Differential. In Stage 1, equivalence class formation occurred for the two experimental groups. In Stage 2, participants of Experimental 1 group achieved the criterion of class reorganization (91\% accuracy) only for symmetry relations; in the other tests, scores were $83 \%$ for six participants. Compared with the Control group, evaluations of the A and D stimuli in the Semantic Differential were partially coherent with the equivalence classes (Experimental 2 group) and with the reversal training and class reorganization tests (Experimental 1 group), with transfer of function for some scales. These results indicate the generality of the present procedures to assess stimulus classes related to professions and gender. However, methodological controls must be improved in relation to pre-experimental history with the stimuli and the quantity of reversed relations.

Key words: stimulus equivalence, class reorganization, transfer of function, delayed matching to sample, semantic differential.

\footnotetext{
O estudo é parte da dissertação de mestrado da primeira autora sob orientação da segunda autora, desenvolvido no Programa de PósGraduação em Ciências do Comportamento do Departamento de Processos Psicológicos Básicos da Universidade de Brasília. Durante a realização do estudo, a primeira autora era bolsista da CAPES. Correspondência relativa a este artigo devem ser enviadas a Alessandra Pinto Rosendo (aleprosendo@gmail.com). O estudo faz parte do programa de pesquisa do Instituto Nacional de Ciência e Tecnologia (INCT-ECCE), com apoio financeiro do CNPq (Processo 573972/2008-7) e da FAPESP (Processo 2008/57705-8).
}

DOI. 10.18542/rebac.v14i1.7157 
$\mathrm{Na}$ Análise do Comportamento, mais especificamente na área de controle de estímulos, a aquisição e manutenção de comportamentos simbólicos envolvidos na linguagem, nos conceitos, estereótipos, preconceitos e no autoconceito, podem ser analisadas a partir da formação de classes de equivalência (de Rose \& Bortoloti, 2007; Watt, Keenan, Bernes, \& Cairns, 1991). Esses comportamentos, que envolvem símbolos sociais, são adquiridos por meio de relações arbitrárias entre símbolos e seus referentes, e variam de acordo com as convenções de cada comunidade verbal (Bortoloti \& de Rose, 2007; de Rose \& Bortoloti, 2007).

Nos estudos sobre equivalência de estímulos são ensinadas, pelo menos, duas discriminações condicionais com um estímulo em comum, em geral, com o procedimento de pareamento ao modelo (do inglês, matching to sample), e são avaliadas a emergência de novas relações, não diretamente treinadas (Albuquerque \& Melo, 2005; Sidman, 1994; Sidman \& Tailby 1982). No contexto social, por exemplo, podem ser aprendidas relações entre profissões (A) e gênero (B) e entre profissões e adjetivos (C) tais como: Enfermeira Feminino e Mecânico - Masculino (AB) e Enfermeira Atenciosa e Mecânico - Grosseiro (AC). A partir dessa história de treino, a emergência de relações de reflexividade entre estímulos fisicamente idênticos (e.g., AA), de simetria que envolve reversão das funções de modelo e comparação dos estímulos (e.g., BA e CA), e de transitividade entre os estímulos que foram relacionados a um estímulo comum (e.g., BC) e equivalência (CB) seriam consideradas evidências da formação de duas classes de equivalência (e.g., Enfermeira, Feminino, Atenciosa; e Mecânico, Masculino, Grosseiro).

Quando classes de equivalência são formadas, funções novas, ou previamente adquiridas por um dos estímulos da classe, podem ser transferidas para outros membros, sem treino adicional (e.g., Bortoloti \& de Rose, 2007; Dougher \& Markham, 1994). Tal possibilidade tem sido considerada como uma alternativa para investigar processos simbólicos envolvidos em fenômenos sociais, como preconceitos e estereótipos relacionados com questões raciais, de gênero e à religião (Carvalho \& de Rose, 2014; Moxon, Keenan, \& Hine, 1993; Watt et al., 1991). Em tais estudos são utilizados estímulos arbitrários (sem significado ou história pré-experimental) e estímulos com significado, estabelecido no contexto social, para verificar o efeito da história pré-experimental na formação de classes de equivalência.

No estudo realizado por Moxon et al. (1993), com estudantes universitários, foram treinadas relações entre palavras impressas referentes a profissões tradicionalmente classificadas como masculinas (motorista de caminhão, executivo e construtor) e sílabas sem sentido, sem referente na língua inglesa (GID, YUM e PUK), e entre sílabas sem sentido e nomes de mulheres (ANNE, KATIE e SUZANNE). No teste de equivalência foi avaliada a emergência de relações entre os nomes de mulheres e profissões masculinas, sendo que, dentre as alternativas de escolha, eram apresentadas duas profissões masculinas e uma feminina (enfermeira, secretaria ou vendedora). A maioria dos participantes (12 dos 19) não formou classes de equivalência, ou seja, as relações de gênero estabelecidas em um contexto extra-experimental competiram com as relações entre estímulos que poderiam derivar das relações treinadas no estudo.

Outros estudos também verificaram que classes de equivalência estabelecidas no ambiente social dificultam a formação de novas relações condicionais, quando essas não são coerentes com a história de aprendizagem pré-experimental (e.g., Carvalho \& de Rose, 2014; Dixon, Rehfeldt, Zlomke, \& Robinson, 2006; Haydu, Camargo, \& Bayer, 2015; Watt et al., 1991). Por outro lado, em uma replicação do estudo de Carvalho e de Rose (2014), Mizael, Almeida, Silveira e de Rose (2016) verificaram a formação de classes de equivalência entre fotografias de faces de pessoas afrodescendentes e símbolos positivos com crianças que avaliaram de maneira negativa tais estímulos antes do estudo. De acordo com os autores, as modificações em variáveis de procedimento, tais como a realização de testes de simetria após os treinos das discriminações condicionais e exposição a tentativas de treino antes dos testes de transitividade e equivalência, favoreceram a formação das classes de equivalência coerentes com o treino e não com a relações préexperimentais.

Dessa forma, variáveis relacionadas com as contingências programadas de treino podem mudar relações entre estímulos estabelecidas no contexto social, as quais dificultam o estabelecimento de classes de equivalência que envolvem novas relações entre estímulos ou a reorganização entre os membros das classes. Nos estudos que investigam a reorganização de classes, em geral, são realizados treino de reversão das discriminações condicionais previamente ensinadas (e.g., Almeida \& de Rose, 2015; Cardoso, 2013; Garotti \& de Rose, 2007).

Portela (2014) investigou a formação e reorganização de classes de equivalência com figuras de silhuetas (Conjunto A - magro, peso normal e obeso), estímulos abstratos (conjuntos - B, C, D) e adjetivos (Conjunto E - Leve, Sadio, Sedentário). Na Etapa 1, estudantes universitários, organizados em Grupo Experimental $(\mathrm{N}=6)$ e Grupo Controle $(\mathrm{N}=5)$, foram expostos a treinos de discriminações condicionais $\mathrm{AC}$ (A1C1, A2C2, A3C3), BC (B1C1, B2C2, B3C3) e CD (C1D1, C2D2, C3D3) e a testes de simetria, transitividade e equivalência (CA, CB, DC; $\mathrm{AB}, \mathrm{AD}, \mathrm{BD}$; BA, DA, DB). $\mathrm{Na}$ Etapa 2, somente o Grupo Experimental foi exposto aos treinos de reversão CDr (C1D2, C2D3, C3D1) e EDr (E1D2, E2D3, E3D1). Foi considerado que as avaliações das silhuetas efetuadas por uma amostra de 90 estudantes indicavam relações pré-experimentais estabelecidas no contexto social entre silhuetas e adjetivos (e.g., silhueta magra e leve; silhueta normal e sadia; e silhueta obesa e sedentária - Treino AE) e, portanto, no Treino EDr eram ensinadas discriminações condicionais diferentes dessas relações pré-experimentais. Por último, nessa segunda etapa foram realizados os testes de reorganização das classes formadas na Etapa 1. O Grupo Controle realizou na Etapa 2 treinos e testes similares aos da Etapa 1 com estímulos familiares (animais, frutas e flores). Para avaliar 
a transferência de função entre os estímulos dos conjuntos A e D, os participantes dos dois grupos foram expostos ao Diferencial Semântico, formado por escalas Likert com adjetivos opostos nas extremidades (padronizado por Almeida, Bortoloti, Ferreira, Schielini, \& de Rose, 2014), em três momentos: no início do estudo, no final da Etapa 1 e após a Etapa 2.

Os resultados do estudo de Portela (2014) mostraram que, na Etapa 1, os participantes dos dois grupos formaram classes de equivalência e que as silhuetas e os estímulos abstratos do Conjunto D foram avaliadas no Diferencial Semântico de maneira similar, o que indica a transferência de função. Entretanto, na Etapa 2 não foi verificada reorganização das classes de equivalência para o Grupo Experimental, uma vez que somente nos testes de simetria o desempenho de quatro dos seis participantes foi coerente com o treino de reversão. Após a Etapa 2, foi verificado no Diferencial Semântico, apenas para os participantes do Grupo Experimental, tendência de avaliações mais positivas da silhueta obesa e mais próximas de zero para a silhueta magra, o que sugere efeito parcial do treino de reversão. De maneira similar ao estudo de Mizael et al. (2016), todos os testes foram precedidos por exposição a tentativas das relações treinadas. No estudo de Portela (2014), no entanto, tal procedimento e o treino de reversão não foram suficientes para modificar as classes de equivalência que eram coerentes com a história pré-experimental.

Estudos anteriores fizeram uso do procedimento de pareamento ao modelo com atraso (do inglês delayed matching to sample - DMTS) e verificaram se ocorria reorganização de classes de equivalência após treinos de reversão, com estímulos abstratos e faces (Almeida e de Rose, 2015; Mizael et al., 2016) ou apenas estímulos abstratos (Garotti e de Rose, 2007; Ribeiro, Silveira, Mackay, \& de Rose, 2016). Esses estudos apresentaram resultados controversos quanto ao uso de DMTS ser mais eficaz do que o pareamento ao modelo simultâneo (simultaneous matching to sample - SMTS) para a formação e reorganização de classes. Entretanto, nessas pesquisas o uso do DMTS possibilitou a reorganização de classes de equivalência e além disso, segundo Almeida e de Rose (2015), o pareamento ao modelo com atraso favorece o estabelecimento de relações mais fortes entre estímulos.

O presente estudo consistiu em uma replicação sistemática da pesquisa de Portela (2014), porém com algumas alterações metodológicas. Foram utilizados estímulos abstratos e estímulos com significado social, relacionados a gênero e profissões, como no estudo de Moxon et al. (1993), porém adaptados ao contexto social brasileiro. Os estímulos (profissões e adjetivos relacionados aos gêneros feminino e masculino) foram definidos a partir de um levantamento realizado anteriormente ao procedimento experimental, com estudantes universitários que não participaram das etapas de treino e teste do estudo. Os treinos e testes foram realizados com o DMTS, uma vez que este procedimento tem sido relacionado com transferência de função mais forte entre os estímulos de classes de equivalência
(Bortoloti \& de Rose, 2012) e com a reorganização de classes (Almeida \& de Rose, 2015). Adicionalmente, os treinos de reversão foram realizados apenas com os estímulos relacionados com as classes das profissões e adjetivos masculino e feminino para favorecer a ocorrência de responder diferencial. Para controlar possíveis efeitos de exposição repetida, os participantes foram expostos ao Diferencial Semântico somente uma vez, após a formação das classes de equivalência ou dos testes de reorganização das classes, e os resultados foram comparados com as avaliações de um grupo controle que não foi exposto a nenhuma das tarefas experimentais programadas.

Em resumo, os objetivos desse estudo consistiram em: (1) investigar o efeito de treinos de reversão, com o procedimento de DMTS, na reorganização de classes de equivalência com palavras (profissões e adjetivos relacionados aos gêneros feminino, masculino ou com ambos) e figuras abstratas; e (2) avaliar a transferência de função entre estímulos com significado social (profissões) e sem significado (estímulos abstratos), após o estabelecimento das classes de equivalência e dos treinos de reversão e testes de reorganização das classes, a partir da aplicação do Diferencial Semântico.

\section{MÉTODO}

\section{Participantes}

Participaram desse estudo 48 estudantes de graduação da Universidade de Brasília (UnB), com idades entre 18 e 33 anos, e que não possuíam história anterior de participação em pesquisas que utilizaram o procedimento de pareamento ao modelo. Dezesseis participantes (oito homens e oito mulheres), matriculados na disciplina Introdução à Psicologia, foram distribuídos aleatoriamente em dois grupos experimentais. Tais estudantes eram procedentes de diferentes cursos de ciências exatas e humanas, tais como: Comunicação Social, Serviço Social, Artes Cênicas, Engenharia Mecânica, Nutrição e Estatística. Os demais 32 participantes, também em número igual de homens e mulheres e de vários cursos de graduação, formaram o Grupo Controle.

A pesquisa foi aprovada pelo Comitê de Ética em Pesquisa do Instituto de Ciências Humanas da Universidade de Brasília (CEP/IH) - CAAE: 66502917.7.0000.5540, Parecer: 2038672. Todos os participantes leram e assinaram o Termo de Consentimento Livre e Esclarecido.

\section{Ambiente e Materiais}

O estudo foi realizado em duas salas localizadas no LIPSI do Instituto de Psicologia da UnB. A sala de coleta de dados individual media aproximadamente $6 \mathrm{~m}^{2} \mathrm{e}$ estava equipada com uma mesa e duas cadeiras. A outra sala, utilizada para a coleta de dados em pequenos grupos, media cerca de $8 \mathrm{~m}^{2}$ e estavam disponíveis 5 mesas e 5 cadeiras. As salas possuíam iluminação artificial e sistema de ar-condicionado.

Foi utilizado um microcomputador equipado com o software Contingência Programada versão 2.0 (Hanna, Batitucci, \& Batitucci, 2014) para a programação das 
tarefas de treino e testes de pareamento ao modelo e registro das respostas de seleção. Também foi utilizada uma escala de Diferencial Semântico, similar a dos estudos de Bortoloti e de Rose (2007) e Portela (2014), para a avaliação do grau de relacionamento entre os estímulos dos conjuntos A e D.

\section{Estímulos}

Foram utilizados 33 estímulos visuais (Figura 1): dois conjuntos de figuras familiares (conjuntos $\mathrm{X}$ e $\mathrm{Y}$ ), dois conjuntos com palavras impressas, sendo um referente a profissões (A) e outro a adjetivos (E), e três conjuntos de figuras abstratas (conjuntos B, C e D). Para os conjuntos B, C e D foram utilizados os mesmos símbolos do estudo de Portela (2014), que foram retirados do estudo de Nalini (2002).

As palavras referentes às profissões e adjetivos (estímulos dos conjuntos A e E) foram selecionadas por meio de levantamento em uma amostra de 202 estudantes universitários (95 homens e 107 mulheres), sendo que metade desses participantes deveria preencher um dentre dois formulários: (1) Seleção de profissões - consistia em uma tabela com 30 profissões e/ou ocupações (e.g., bancário(a), assistente social, professor(a), economista) que deveriam ser classificadas como tipicamente relacionadas a cada gênero (masculino e feminino) ou com ambos; e (2) Seleção de adjetivos - era composto por uma lista de 40 adjetivos (e.g., racional, competente, egoísta), apresentados de maneira semi-randômica; a tarefa do participante consistia em avaliar e assinalar apenas os cinco adjetivos que considerasse mais relacionados com três profissões, as quais foram selecionadas pela outra metade dos participantes como as atribuídas a cada gênero ou ambos com maior frequência. As listas de profissões e de adjetivos foram confeccionadas a partir de consultas a dados do Censo do Ensino Superior de 2012 e a sites de listas de adjetivos que descrevem pessoas e/ou características de personalidade.

\begin{tabular}{|c|c|c|c|c|c|c|c|c|c|}
\hline \multirow{2}{*}{ Estímulos } & \multicolumn{2}{|c|}{ Figuras Geométricas } & \multirow{2}{*}{\multicolumn{2}{|c|}{$\begin{array}{c}\text { Profissões } \\
\text { A }\end{array}$}} & \multicolumn{3}{|c|}{ Figuras Abstratas } & \multirow{2}{*}{\multicolumn{2}{|c|}{$\begin{array}{c}\text { Adjetivos } \\
\text { E }\end{array}$}} \\
\hline & $\mathrm{X}$ & $\mathrm{Y}$ & & & B & $\mathrm{C}$ & $\mathrm{D}$ & & \\
\hline 1 & & & $\begin{array}{c}\text { Trabalhadora Doméstica } \\
\text { Secretária } \\
\text { Assistente Social }\end{array}$ & Ca $\begin{array}{l}\mathrm{A}^{1} \\
\mathrm{~A} 1^{2} \\
\mathrm{~A} 1^{3}\end{array}$ & & & X & $\begin{array}{c}\text { Organizada } \\
\text { Mal Remunerada } \\
\text { Desvalorizada }\end{array}$ & $\begin{array}{l}\mathrm{E} 1^{1} \\
\mathrm{E} 1^{2} \\
\mathrm{E} 1^{3}\end{array}$ \\
\hline 2 & & & $\begin{array}{l}\text { Comerciante } \\
\text { Vendedor(a) } \\
\text { Advogado(a) }\end{array}$ & $\begin{array}{l}\mathrm{A} 2^{1} \\
\mathrm{~A} 2^{2} \\
\mathrm{~A} 2^{3}\end{array}$ & CA & $7: 0$ & 出 & $\begin{array}{l}\text { Competitivo(a) } \\
\text { Sociável } \\
\text { Ambicioso(a) }\end{array}$ & $\begin{array}{l}\mathrm{E} 2^{1} \\
\mathrm{E} 2^{2} \\
\mathrm{E} 2^{3}\end{array}$ \\
\hline 3 & & & $\begin{array}{l}\text { Mecânico } \\
\text { Pedreiro } \\
\text { Marceneiro }\end{array}$ & $\begin{array}{l}\mathrm{A} 3^{1} \\
\mathrm{~A} 3^{2} \\
\mathrm{~A} 3^{3}\end{array}$ & & & 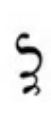 & $\begin{array}{c}\text { Competente } \\
\text { Ativo } \\
\text { Cauteloso }\end{array}$ & $\begin{array}{l}\mathrm{E}^{1} \\
\mathrm{E}^{2} \\
\mathrm{E} 3^{3}\end{array}$ \\
\hline
\end{tabular}

Figura 1. Conjuntos de estímulos que foram utilizados no estudo. Os números 1, 2 e 3 sobrescritos indicam as variações de cada profissão (Conjunto A) de acordo com o gênero ou dos adjetivos relacionados com as profissões (Conjunto E).

\section{Procedimento}

Os participantes foram distribuídos aleatoriamente em três grupos: Grupo Experimental $1(\mathrm{~N}=$ 8), Grupo Experimental $2(\mathrm{~N}=8)$ e Grupo Controle $(\mathrm{N}=$ 32). Os grupos experimentais se diferenciavam pela exposição ao treino de reversão de duas discriminações condicionais após a formação das classes de equivalência. O Grupo Controle participou apenas da avaliação da transferência de função.

Os dois grupos experimentais foram expostos ao Pré-Treino da tarefa de discriminação condicional e a uma sequência de treinos e testes para a formação das classes de equivalência (Etapa 1), composta por: Pré-Treino; Treinos AC, BC e CD; Treino Misto e Testes de Formação de Classes de Equivalência. Somente o Grupo Experimental 1 foi exposto a Etapa 2 de reorganização de classes, formada por: Treino CDr e EDr; Treino Misto e Testes de Reorganização de Classes. As relações treinadas no Treino EDr eram diferentes das relações que os estudantes, que participaram apenas da etapa de seleção dos estímulos, estabeleceram entre profissões relacionadas a gêneros e adjetivos, as quais foram também consideradas como aprendidas pré-experimentalmente no contexto social pelos participantes do presente estudo. Adicionalmente, o Treino EDr está relacionado com a emergência de $\mathrm{AD}$, após os treinos $\mathrm{AC}$ e $\mathrm{CD}$ realizados na Etapa 1. Ao final da Etapa 1 (Grupo Experimental 2) e da Etapa 2 (Grupo Experimental 1), foi aplicado o Diferencial Semântico para a avaliação da transferência de função.

Nas tarefas de treino e teste das Etapas 1 e 2 foi utilizado o procedimento DMTS com estímulos visuais. Em todas as tentativas de treino era apresentado um 
estímulo modelo e o participante era solicitado a clicar nele com o uso do mouse. Tal resposta resultava na remoção do estímulo modelo e após $1,5 \mathrm{~s}$ eram apresentados os estímulos de comparação. A tarefa do participante consistia em selecionar o estímulo correspondente ao modelo previamente apresentado. As respostas corretas resultavam na apresentação da figura de um smile (carinha feliz) simultaneamente com estímulos auditivos (e.g. "Ótimo!", "Muito bem!"). Posteriormente, era apresentado o Intervalo entre Tentativas (IET) de 1,5 s, o qual era seguido por uma nova tentativa. As respostas incorretas resultavam na apresentação de uma tela com a figura de um "X vermelho", seguida do IET e depois por uma nova tentativa (Figura 2). Nas tentativas de teste não foram apresentadas consequências diferenciais para respostas corretas e incorretas.

A Tabela 1 apresenta a sequência dos treinos e testes realizados com os grupos experimentais nas Etapas 1 e 2, o número mínimo de tentativas para cada sessão de treino ou por blocos dos testes, os critérios de aprendizagem exigidos por bloco e as discriminações condicionais treinadas ou testadas. As características dos procedimentos de treino e teste das Etapas 1 e 2 serão descritas a seguir.

Tabela 1

Sequência de Treinos e Testes Realizados na Etapa 1 e na Etapa 2, Número de Tentativas, Critério de Aprendizagem e Discriminações Condicionais Treinadas ou Testadas.

\begin{tabular}{|c|c|c|c|}
\hline Treinos e Testes & $\begin{array}{l}\text { Número de } \\
\text { Tentativas }^{\text {a }}\end{array}$ & $\begin{array}{c}\text { Critério de } \\
\text { Aprendizagem }\end{array}$ & Discriminações Condicionais \\
\hline \multicolumn{4}{|c|}{ Etapa 1 - Formação de Classes de Equivalência } \\
\hline Pré-Treino XY & 24 & $100 \%$ & $\mathrm{X} 1 \mathrm{Y} 1, \mathrm{X} 2 \mathrm{Y} 2$ e X3Y3 \\
\hline Treino AC & 42 & $100 \%$ & $\mathrm{~A} 1{ }^{\mathrm{b}} \mathrm{C} 1, \mathrm{~A} 2^{\mathrm{b}} \mathrm{C} 2$ e $\mathrm{A} 3^{\mathrm{b}} \mathrm{C} 3$ \\
\hline Treino BC & 42 & $100 \%$ & $\mathrm{~B} 1 \mathrm{C} 1, \mathrm{~B} 2 \mathrm{C} 2$ e $\mathrm{B} 3 \mathrm{C} 3$ \\
\hline Treino CD & 42 & $100 \%$ & $\mathrm{C} 1 \mathrm{D} 1, \mathrm{C} 2 \mathrm{D} 2$ e $\mathrm{C} 3 \mathrm{D} 3$ \\
\hline Treino Misto AC/BC/CDr & 63 & $100 \%$ & $\begin{array}{c}\mathrm{A} 1^{\mathrm{b}} \mathrm{C} 1, \mathrm{~A} 2^{\mathrm{b}} \mathrm{C} 2, \mathrm{~A} 3^{\mathrm{b}} \mathrm{C} 3, \mathrm{~B} 1 \mathrm{C} 1, \\
\mathrm{~B} 2 \mathrm{C} 2, \mathrm{~B} 3 \mathrm{C} 3, \mathrm{C} 1 \mathrm{D} 1, \mathrm{C} 2 \mathrm{D} 2, \mathrm{C} 3 \mathrm{D} 3\end{array}$ \\
\hline Teste de Simetria & 9 & $88 \%$ & $\mathrm{CA}^{\mathrm{b}}, \mathrm{CB}$ e $\mathrm{DC}$ \\
\hline Teste de Transitividade & 9 & $88 \%$ & $A^{b} B, A^{b} D$ e $B D$ \\
\hline Teste de Equivalência & 9 & $88 \%$ & $\mathrm{BA}^{\mathrm{b}}, \mathrm{DA}^{\mathrm{b}}$ e $\mathrm{DB}$ \\
\hline \multicolumn{4}{|c|}{ Etapa 2 - Reorganização de Classes de Equivalência } \\
\hline Treino CDr & 42 & $100 \%$ & C1D3, C2D2, C3D1 \\
\hline Treino EDr & 42 & $100 \%$ & $\mathrm{E} 1^{\mathrm{b}} \mathrm{D} 3, \mathrm{E} 2^{\mathrm{b}} \mathrm{D} 2, \mathrm{E} 3^{\mathrm{b}} \mathrm{D} 1$ \\
\hline $\begin{array}{l}\text { Treino Misto } \\
\text { AC/BC/CDr/EDr }\end{array}$ & 84 & $100 \%$ & $\begin{array}{c}\mathrm{A} 1^{\mathrm{b}} \mathrm{C} 1, \mathrm{~A} 2^{\mathrm{b}} \mathrm{C} 2, \mathrm{~A} 3^{\mathrm{b}} \mathrm{C} 3, \mathrm{~B} 1 \mathrm{C} 1, \\
\mathrm{~B} 2 \mathrm{C} 2, \mathrm{~B} 3 \mathrm{C} 3, \mathrm{C} 1 \mathrm{D} 3, \mathrm{C} 2 \mathrm{D} 2, \mathrm{C} 3 \mathrm{D} 1, \\
\mathrm{E} 1^{\mathrm{b}} \mathrm{D} 3, \mathrm{E} 2^{\mathrm{b}} \mathrm{D} 2, \mathrm{E} 3^{\mathrm{b}} \mathrm{D} 1\end{array}$ \\
\hline Teste de Simetria & 15 & $91 \%$ & $\mathrm{CA}^{\mathrm{b}}, \mathrm{CB}, \mathrm{DCr}$ e $\mathrm{DE}^{\mathrm{b}} \mathrm{r}$ \\
\hline Teste de Transitividade & 12 & $91 \%$ & $A^{b} D, A^{b} B, B D, C E^{b} r$ \\
\hline Teste de Equivalência & 12 & $91 \%$ & $\mathrm{DA}^{\mathrm{b}}, \mathrm{BA}^{\mathrm{b}}, \mathrm{DB}$ e $\mathrm{E}^{\mathrm{b}} \mathrm{Cr}$ \\
\hline
\end{tabular}

Nota. ${ }^{a}$ Número mínimo de tentativas por sessão dos treinos ou número de tentativas por bloco de teste; ${ }^{\mathrm{b}}$ Apresentação de uma das três variações de cada elemento dos conjuntos A e E, conforme Figura 1.

Pré-Treino de discriminações condicionais. A sessão de Pré-Treino era constituída por 24 tentativas de DMTS distribuídas em oito blocos, com as figuras geométricas dos conjuntos X e Y (Figura 1). Foi utilizado como critério de finalização $100 \%$ de acerto no último bloco. Caso houvesse erro em algum bloco, este era repetido até três vezes, no máximo. Caso o critério de $100 \%$ de acerto não fosse atingido em um bloco na terceira tentativa ou caso houvesse algum erro no último bloco, o Pré-Treino era encerrado e repetido na próxima sessão.

Treino das discriminações condicionais. Cada sessão de treino foi composta por, no mínimo, 42 tentativas organizadas em 13 blocos, com uma a oito tentativas por bloco. As três discriminações condicionais, de cada treino ( $\mathrm{AC}, \mathrm{BC}$ e $\mathrm{CD}$ ) eram ensinadas uma por 
vez (e.g., A1C1, A2C2 e A3C3) e, posteriormente, misturadas em um mesmo bloco.

No Treino AC, todos os nove estímulos do Conjunto A (Profissões) foram apresentados de acordo com as três possibilidades de profissões relacionadas com os gêneros feminino, masculino ou com ambos. A profissão feminina (A1), por exemplo, variava dentre três opções: TRABALHADORA DOMÉSTICA (A1 $\left.{ }^{1}\right)$, SECRETÁRIA (A12) e ASSISTENTE SOCIAL (A1 ${ }^{3}$ ); o mesmo ocorria para as profissões masculinas ou relacionadas com os dois gêneros (conforme Figura 1).

Após o Treino CD, foi realizado o Treino Misto $\mathrm{AC} / \mathrm{BC} / \mathrm{CD}$, o qual era composto por 45 tentativas organizadas em seis blocos. Nos cinco primeiros blocos $100 \%$ das respostas corretas eram reforçadas. No bloco final, o reforço era apresentado para $50 \%$ das respostas corretas, com o objetivo de preparar os participantes para os testes que eram realizados em extinção.

Nas sessões de treino das discriminações condicionais individuais e no Treino Misto, para avançar de um bloco de tentativas para o próximo era necessário atingir o critério de $100 \%$ de acerto. Em caso de erros, cada bloco podia ser repetido por, no máximo, três vezes. Se até a terceira repetição o critério não fosse atingido, a sessão era encerrada e o treino era repetido na sessão seguinte. O critério de finalização de todos os treinos era de $100 \%$ de acerto no bloco final.

Teste de Formação de Classes de Equivalência. A sessão de teste era organizada em cinco blocos que continham nove tentativas cada, sendo dois blocos de Treino Misto AC/BC/CD (um com reforço para todas as respostas corretas e outro com reforço para $50 \%$ dos acertos) e três blocos de teste, realizados em extinção, para avaliar separadamente as relações de Simetria, Transitividade e de Equivalência. No teste de simetria foram avaliadas as relações $\mathrm{CA}, \mathrm{CB}$ e $\mathrm{DC}$; no de transitividade, as relações $\mathrm{AB}, \mathrm{AD}$ e $\mathrm{BD}$ eram testadas e, por último, as relações de equivalência (BA, DA e DB) foram testadas. A formação de classes de equivalência foi avaliada de acordo com o critério de $88 \%$ de acerto em cada bloco de teste (um erro em nove tentativas). Caso esse critério não fosse atingido, o participante repetia a sessão de Treino Misto e depois a sessão de teste por até três vezes, no máximo. Se após três repetições da sessão de teste a porcentagem de acerto se mantivesse inferior ao critério estabelecido, a participação do estudante era encerrada.

Treino de Reversão das discriminações condicionais. Foram realizados os treinos de reversão das discriminações condicionais CD (CDr) e ED (EDr). No Treino CDr foi reforçada a escolha de D3 na presença do modelo $\mathrm{C} 1$ e de $\mathrm{D} 1$ na presença do modelo $\mathrm{C} 3$. A relação C2D2 não foi modificada em relação ao Treino CD original.

No Treino EDr foram treinadas duas relações revertidas entre os adjetivos relacionados à profissão feminina (E1) e o símbolo D3 (classe de profissões masculinas) e os adjetivos relacionados à profissão masculina (E3) e o símbolo D1 (classe de profissões femininas). Não foi revertida a relação entre os adjetivos e estímulos abstratos relacionados aos dois gêneros (E2D2). Assim como no Treino AC, no Treino EDr todos os estímulos do Conjunto $\mathrm{E}$ foram apresentados, de acordo com as três possibilidades de adjetivos relacionados com as profissões mais representativas dos gêneros feminino, masculino ou com ambos. $\mathrm{O}$ adjetivo relacionado com a profissão feminina (E1), por exemplo, podia variar dentre três opções: ORGANIZADA (E1 $\left.{ }^{1}\right)$, MAL REMUNERADA (E1 $\left.{ }^{2}\right)$ e DESVALORIZADA (E1 $\left.1^{3}\right)$, conforme Figura 1.

Após os treinos CDr e EDr foi realizado o Treino Misto AC/BC/CDr/EDr, que era composto por 60 tentativas organizadas em sete blocos. Os critérios de repetição de blocos e de sessões, e de finalização dos treinos das relações e do Treino Misto com reversão eram similares aos dos treinos sem reversão.

Teste de Reorganização de Classes de Equivalência. Esse teste era similar ao da Etapa 1, e foi composto por 63 tentativas, organizadas em cinco blocos, sendo que os três blocos de teste eram precedidos por dois blocos de Treino Misto das discriminações condicionais $\mathrm{AC} / \mathrm{BC} / \mathrm{CDr} / \mathrm{EDr}$, um com $100 \%$ de apresentação de reforços e outro com reforço para metade das tentativas. Exceto o bloco de teste de simetria, que tinha 15 tentativas, todos os outros blocos possuíam 12 tentativas. No bloco de Teste de Simetria eram avaliadas as relações CA, CB, DCr e DEr, no Teste de Transitividade as relações $\mathrm{AD}, \mathrm{AB}, \mathrm{BD}, \mathrm{CEr}$, e no Teste de Equivalência as relações DA, BA, DB e ECr. O critério para considerar que as classes foram reorganizadas foi de, no mínimo, $91 \%$ de acerto ( 1 erro em 12 ou 15 tentativas) em cada bloco de teste. Caso o critério nos blocos de teste não fosse atingido, o participante era submetido à sessão anterior de Treino Misto e, posteriormente, os testes de reorganização eram repetidos. Esse procedimento podia ser realizado até duas vezes, o que resultaria em um total de três exposições aos blocos de teste de reorganização.

Avaliação da Transferência de Função. Foi aplicado o Diferencial Semântico com o objetivo de verificar como os estímulos do Conjunto D (D1, D2 e D3) e três do Conjunto A (profissões: Trabalhadora Doméstica, Comerciante e Mecânico) eram avaliados nas 13 escalas bipolares pelos grupos Controle, Experimental 2 (exposto apenas a Etapa 1, de formação de classes de equivalência) e Experimental 1 (exposto aos treinos de reversão na Etapa 2).

O Diferencial Semântico foi aplicado individualmente para os participantes dos grupos experimentais e em pequenos grupos, de até cinco participantes, para o Grupo Controle. Eram fornecidas uma folha com instruções e seis folhas com as escalas de Diferencial Semântico. O estímulo a ser avaliado ficava na parte superior da folha e, logo abaixo, estavam disponíveis 13 escalas, sendo que cada uma dessas escalas compreendia sete intervalos com adjetivos opostos nas extremidades (para uma revisão, consultar Bortoloti \& de 
Rose, 2009). A tarefa consistia em marcar um " $x$ " na posição da escala que, de acordo com a avaliação do participante, melhor representasse a relação entre o estímulo apresentado e cada par de adjetivos opostos. A posição do " $x$ " assinalado pelo participante foi utilizada como indicador da relação do estímulo com os adjetivos.

\section{RESULTADOS}

Pré-Treino e Treino das discriminações condicionais. No Pré-Treino, dos 16 participantes dos dois grupos experimentais, nove atingiram o critério em apenas uma sessão, seis foram expostos a duas sessões, e somente um (P5) foi submetido a três exposições. Na Etapa 1, os desempenhos dos participantes dos Grupos Experimentais 1 e 2 foram precisos nos Treinos das relações $\mathrm{AC}, \mathrm{BC}$ e $\mathrm{CD}$, com nenhum ou poucos erros (16), exceto para P15 (14 erros), e 10 participantes foram expostos a apenas uma sessão de cada uma das relações condicionais treinadas.

Para a análise dos desempenhos no Treino Misto $\mathrm{AC} / \mathrm{BC} / \mathrm{CD}$ da Etapa 1 foram consideradas as tentativas do Treino Misto somadas às tentativas dos blocos de treino misto que precediam os blocos de teste da sessão de Teste de Formação de Classes de Equivalência. Nenhum ou poucos erros (1-5) foram verificados para 10 participantes dos dois grupos experimentais.

Nos treinos das relações CDr e EDr da Etapa 2, o desempenho dos participantes do Grupo Experimental 1 também foi preciso, com nenhum ou poucos erros (15). Para a análise dos dados do Treino Misto
AC/BC/CDr/EDr da Etapa 2 foram consideradas as tentativas de treino misto incluídas na sessão do Teste de Reorganização de Classes. Os participantes do Grupo Experimental 1 apresentaram mais erros (6-22) no Treino Misto da Etapa 2 do que no Treino Misto da Etapa 1.

Testes de Formação e de Reorganização de Classes. A Tabela 2 apresenta a quantidade de acerto por total de tentativas nos testes de formação de classes (Etapa 1) e de reorganização de classes (Etapa 2). A letra a indica que ocorreu mais de uma exposição ao teste da Etapa 1; a letra ${ }^{\mathrm{b}}$ destaca os participantes que foram expostos três vezes aos testes de reorganização por não atingirem o critério mínimo de acerto em cada bloco; e a letra ${ }^{c}$ indica erro de procedimento para três participantes do Grupo Experimental 1 que não atingiram o critério na segunda exposição aos testes de reorganização e não passaram por uma terceira exposição.

No Teste de Formação de Classes (parte superior da Tabela 2), a maioria dos participantes apresentou desempenho preciso após uma única exposição aos blocos de teste de simetria, transitividade e equivalência. Considerando o critério estabelecido (no máximo, um erro em cada bloco de teste), esses resultados demonstram que, para 13 participantes, houve formação de três classes de equivalência com quatro elementos (A1B1C1D1, A2B2C2D2 e A3B3C3D3) na primeira exposição aos testes. Outros três participantes do Grupo Experimental 2 (P11, P15 e P16) atingiram o critério de formação de classes de equivalência na segunda exposição aos testes.

\section{Tabela 2}

Quantidade de Acerto por Total de Tentativas, nos Blocos de Simetria, Transitividade e Equivalência dos Testes de Formação de Classes de Equivalência (Etapa 1) e Reorganização de Classes de Equivalência (Etapa 2)

\begin{tabular}{|c|c|c|c|c|c|c|c|c|}
\hline \multicolumn{9}{|c|}{ Teste de Formação de Classes de Equivalência (Etapa 1) } \\
\hline \multirow{2}{*}{ Testes } & \multicolumn{8}{|c|}{ Grupo Experimental 1} \\
\hline & $\mathrm{P} 1$ & $\mathrm{P} 2$ & $\mathrm{P} 3$ & $\mathrm{P} 4$ & P5 & P6 & P7 & P8 \\
\hline Simetria & $8 / 9$ & $9 / 9$ & $8 / 9$ & $9 / 9$ & $9 / 9$ & $9 / 9$ & $9 / 9$ & $9 / 9$ \\
\hline Transitividade & $9 / 9$ & $9 / 9$ & $9 / 9$ & $9 / 9$ & $9 / 9$ & $9 / 9$ & $9 / 9$ & $8 / 9$ \\
\hline \multirow[t]{3}{*}{ Equivalência } & $9 / 9$ & $9 / 9$ & $9 / 9$ & $9 / 9$ & $9 / 9$ & $9 / 9$ & $9 / 9$ & $9 / 9$ \\
\hline & \multicolumn{8}{|c|}{ Grupo Experimental 2} \\
\hline & $\mathrm{P9}$ & $\mathrm{P} 10$ & P11 & $\mathrm{P} 12$ & $\mathrm{P} 13$ & $\mathrm{P} 14$ & $\mathrm{P} 15$ & P16 \\
\hline Simetria & $9 / 9$ & $9 / 9$ & $16 / 18^{\mathrm{a}}$ & $9 / 9$ & $9 / 9$ & $8 / 9$ & $18 / 18^{\mathrm{a}}$ & $18 / 18^{\mathrm{a}}$ \\
\hline Transitividade & $9 / 9$ & $9 / 9$ & $13 / 18^{\mathrm{a}}$ & $9 / 9$ & $8 / 9$ & $9 / 9$ & $12 / 18^{\mathrm{a}}$ & $18 / 18^{\mathrm{a}}$ \\
\hline Equivalência & $9 / 9$ & $9 / 9$ & $15 / 18^{\mathrm{a}}$ & $9 / 9$ & $8 / 9$ & $9 / 9$ & $13 / 18^{\mathrm{a}}$ & $14 / 18^{\mathrm{a}}$ \\
\hline \multicolumn{9}{|c|}{ Teste de Reorganização de Classes de Equivalência (Etapa 2) } \\
\hline \multirow{2}{*}{ Testes } & \multicolumn{8}{|c|}{ Grupo Experimental 1} \\
\hline & $\mathrm{P} 1$ & $\mathrm{P} 2$ & $\mathrm{P} 3$ & $\mathrm{P} 4$ & P5 & P6 & $\mathrm{P} 7$ & $\mathrm{P} 8$ \\
\hline Simetria & $44 / 45^{\mathrm{b}}$ & $30 / 30^{c}$ & $45 / 45^{\mathrm{b}}$ & $44 / 45^{\mathrm{b}}$ & $30 / 30^{c}$ & $45 / 45^{b}$ & $30 / 30^{c}$ & $44 / 45^{\mathrm{b}}$ \\
\hline Transitividade & $25 / 36^{\mathrm{b}}$ & $20 / 24^{c}$ & $30 / 36^{\mathrm{b}}$ & $30 / 36^{\mathrm{b}}$ & $20 / 24^{c}$ & $29 / 36^{\mathrm{b}}$ & $20 / 24^{c}$ & $30 / 36^{\mathrm{b}}$ \\
\hline Equivalência & $22 / 36^{\mathrm{b}}$ & $20 / 24^{c}$ & $30 / 36^{\mathrm{b}}$ & $30 / 36^{\mathrm{b}}$ & $20 / 24^{c}$ & $29 / 36^{\mathrm{b}}$ & $20 / 24^{c}$ & $30 / 36^{\mathrm{b}}$ \\
\hline
\end{tabular}

Nota. ${ }^{\text {a }}$ Critério do teste não atingido na primeira exposição. ${ }^{\mathrm{b}}$ Participantes que repetiram a sessão de teste de reorganização três vezes.

${ }^{\mathrm{c}}$ Erro de procedimento: Duas exposições aos testes de reorganização. 
No Teste de Reorganização de Classes da Etapa 2 (parte inferior da Tabela 2), todos os participantes repetiram o teste, pois nenhum deles atingiu o critério estabelecido em cada um dos três blocos (máximo de um erro por bloco). Porém os participantes P2, P5 e P7 foram expostos à somente uma repetição adicional dos blocos do teste de reorganização, devido a erro de procedimento.

De acordo com a Tabela 1 , todos os participantes do Grupo Experimental 1 atingiram o critério de reorganização de classes no bloco de teste de simetria. Nos testes de transitividade e equivalência, a maior quantidade de erros foi verificada para o participante P1, com 11 erros nos testes de transitividade e 14 erros nos testes de equivalência. Para seis participantes (P2, P3, P4, P5, P7 e P8) ocorreram dois erros em cada exposição aos blocos de teste de transitividade e de equivalência. Já o participante P6 cometeu sete erros em cada um dos testes de transitividade e de equivalência. Assim, os resultados mostram que, apesar dos participantes repetirem os testes de reorganização de classes, eles continuaram cometendo os mesmos erros nos blocos que verificavam as relações de transitividade e de equivalência.

Avaliação da Transferência de Função a partir do Diferencial Semântico. Foram analisados os resultados das avaliações dos três estímulos do Conjunto D (D1, D2 e D3) realizadas pelos grupos experimentais 1 e 2, e das avaliações de três estímulos do Conjunto A (Trabalhadora Doméstica, Comerciante e Mecânico) do Grupo Controle. Foram atribuídos aos sete intervalos das escalas os valores $-3,-2,-1,0,+1,+2$ e +3 . Para cada grupo, foi calculada a mediana das avaliações em cada escala, a qual corresponde ao valor central dentre as avaliações dos participantes. Serão realizadas descrições das avaliações a partir de inspeção visual e análises estatísticas.

A Figura 2 (parte superior) apresenta as comparações entre as medianas das avaliações do estímulo A1 ${ }^{1}$ (Trabalhadora Doméstica) realizadas pelos participantes do Grupo Controle com as avaliações do estímulo D3 realizadas pelos participantes do Grupo Experimental 1 (que foi exposto aos treinos de reversão: C1D3, C3D1 e E1D3, E3D1) e do estímulo D1 realizadas pelos participantes do Grupo Experimental 2, gráficos à esquerda e à direita, respectivamente.

Verifica-se que a avaliação do estímulo D3 (originalmente da classe masculina, mas posteriormente treinado com um elemento da classe feminina) feita pelos participantes do Grupo Experimental 1 (Figura 2, lado superior esquerdo) diferiu da avaliação de $\mathrm{A} 1^{1}$ realizada pelos participantes do Grupo Controle. A avaliação do estímulo $\mathrm{A} 1^{1}$ nas 13 escalas variou de -2 à +2 , sendo verificado valores positivos apenas nas escalas LENTORÁPIDO e PASSIVO-ATIVO, e valores neutros ou negativos nas outras 11 escalas. Já na avaliação do estímulo D3 pelo Grupo Experimental 1, os valores das medianas foram mais positivos e variaram entre os valores de 0 e +1 , sendo a exceção o valor negativo na escala SUBMISSO-DOMINANTE.

Para o Grupo Experimental 2 (Figura 2, lado superior direito), que foi exposto apenas aos treinos e testes de formação de classes de equivalência, as medianas das avaliações do estímulo D1 (da classe feminina) tenderam a ser similares às medianas das avaliações do Grupo Controle para o estímulo $\mathrm{A}^{1}{ }^{\mathbf{1}}$, exceto nas últimas quatro escalas, nas quais as medianas das avaliações do Grupo Experimental 2 foram zero e para o Grupo Controle foram obtidos valores positivos nas escalas LENTORÁPIDO e PASSIVO-ATIVO e negativos nas escalas POBRE-RICO E SUBMISSO-DOMINANTE.

O Teste de Mann-Whitney mostrou diferenças significativas entre as avaliações de $\mathrm{A} 1^{1}$ pelo Grupo Controle e de D3 pelo Grupo Experimental 1 nas escalas Tenso-Relaxado $(\mathrm{U}=55,000 ; p<.05)$, Pesado-Leve $(\mathrm{U}=$ 54,500; $p<.05)$, Duro-Macio ( $\mathrm{U}=55,000 ; p<.05) \mathrm{e}$ Lento-Rápido $(\mathrm{U}=65,500 ; p<.05)$ e entre as avaliações de A1 ${ }^{1}$ pelo Grupo Controle e de D1 pelo Grupo Experimental 2 nas escalas Lento-Rápido ( $\mathrm{U}=54,000 ; p<$ $.05)$, Pobre-Rico $(\mathrm{U}=29,500 ; p<.01)$ e SubmissoDominante $(\mathrm{U}=34,000 ; p<.01)$.

As comparações das avaliações do estímulo A2 ${ }^{1}$ (Comerciante) realizada pelo Grupo Controle e do estímulo D2 realizadas pelos grupos experimentais 1 e 2 são apresentadas na parte central da Figura 2. Os estímulos A2 ${ }^{1}$ e D2 pertenciam a mesma classe, relacionada com os dois gêneros, e as relações entre os membros dessa classe foram mantidas de acordo com os treinos originais da Etapa 1 em todo o estudo.

Verifica-se que as medianas das avaliações do estímulo A2 ${ }^{1}$ realizadas pelo Grupo Controle apresentaram, em sua maioria, valores nulos ou positivos (variação de $0 \mathrm{a}+1$ ), exceto o valor negativo obtido na escala TENSO-RELAXADO. As avaliações do estímulo D2 do Grupo Experimental 1 foram mais positivas do que as avaliações do estímulo A2 ${ }^{1}$ realizadas pelo Grupo Controle (Figura 2, parte central esquerda), com valores positivos nas medianas de 10 escalas, nulo em duas, e negativo apenas na escala PESADO-LEVE. Para o Grupo Experimental 2 (Figura 2, parte central direita), as medianas das avaliações do estímulo D2 também tenderam a ser mais positivas do que as avaliações do estímulo A2 ${ }^{1}$ feitas pelo Grupo Controle, exceto nas escalas PESADOLEVE e DURO-MACIO, nas quais os valores das medianas foram negativos.

O Teste de Mann-Whitney mostrou que não houve diferença significativa entre as avaliações dos grupos Controle e Experimental 1. Entretanto, diferenças significativas foram verificadas entre as avaliações de A2 e D2 realizadas pelos grupos Controle e Experimental 2 nas escalas Tenso-Relaxado ( $\mathrm{U}=65,000 ; p<.05)$, DuroMacio $(\mathrm{U}=45,000 ; p<.05)$ e Passivo-Ativo $(\mathrm{U}=60,500$; $p<.05)$. 


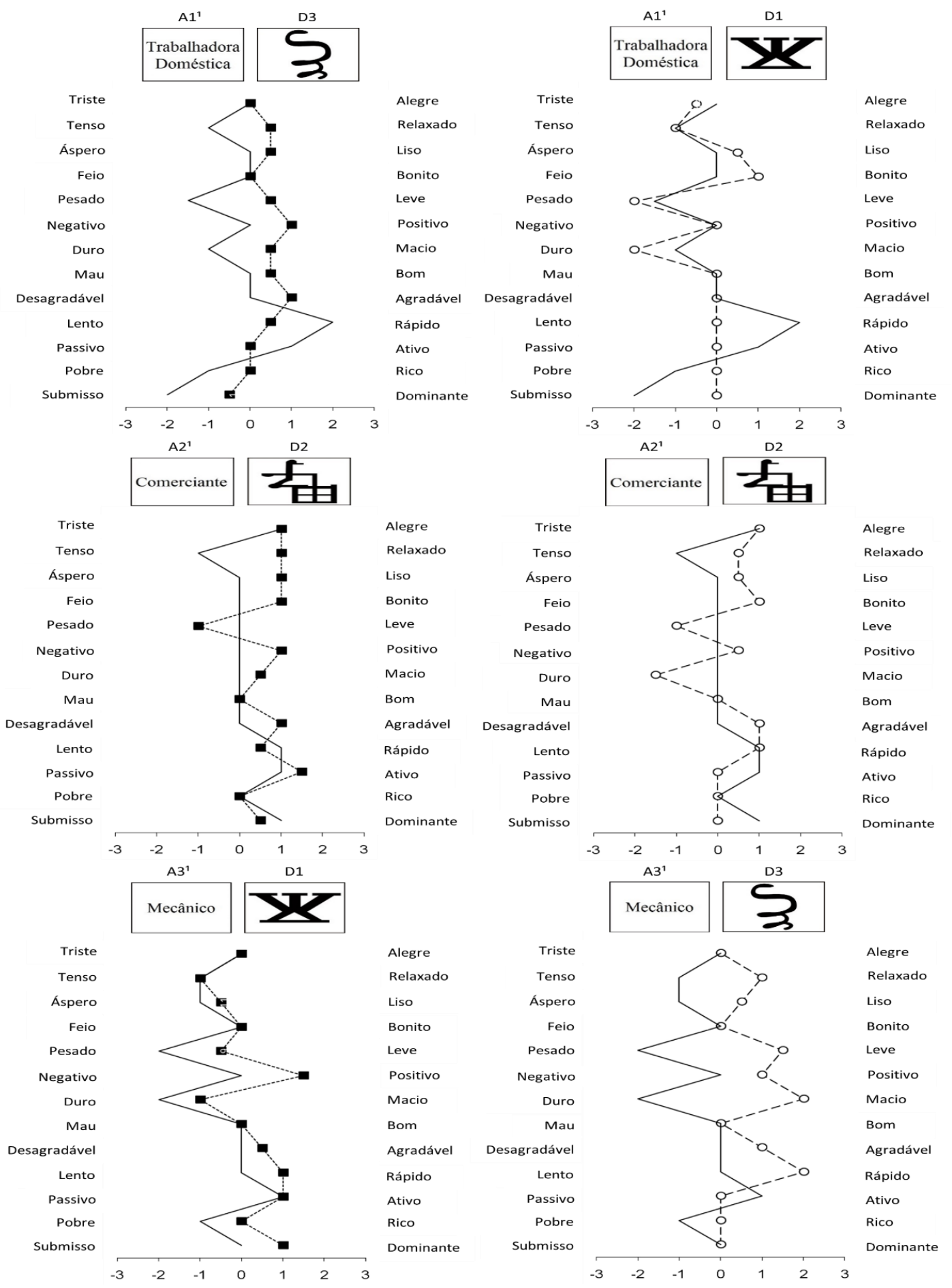

Figura 2. Medianas das avaliações em cada uma das 13 escalas do Diferencial Semântico dos estímulos A1 ${ }^{1}$ (Trabalhadora Doméstica), A2 ${ }^{1}$ (Comerciante) e A3 ${ }^{1}$ (Mecânico) realizadas pelos participantes do Grupo Controle (linhas pretas contínuas); dos estímulos D3, D2 e D1 pelos participantes do Grupo Experimental 1 (gráficos à esquerda; linhas pontilhadas com quadrados preenchidos) e dos estímulos D1, D2 e D3 pelos participantes do Grupo Experimental 2 (gráficos à direita; linhas tracejadas com círculos)

A Figura 2 (parte inferior) apresenta as comparações das avaliações do estímulo A31 (Mecânico) realizadas pelo Grupo Controle, com as avaliações do estímulo D1 do Grupo Experimental 1 (devido ao treino de reversão) e do estímulo D3 realizadas pelos participantes do Grupo Experimental 2. As medianas das avaliações do Grupo Experimental 1 para o estímulo D1 (Figura 2, parte inferior esquerda) foram positivas ou neutras, exceto em quatro escalas (TENSO-RELAXADO, ÁSPERO-LISO, PESADO-LEVE e DURO-MACIO), nas quais os valores 
apresentados foram negativos, o que diferiu das medianas das avaliações do Grupo Controle para a profissão masculina (A3 $\left.{ }^{1}\right)$, que foi positiva somente na escala PASSIVO-ATIVO. Para o Grupo Experimental 2 (Figura 2, parte inferior direita), as medianas das avaliações do estímulo D3 foram positivas ou neutras, com variação de 0 $a+2$. Tais avaliações diferiram das avaliações do estímulo A $3^{1}$ realizadas pelo Grupo Controle, com valor positivo apenas na mediana da escala PASSIVO-ATIVO.

O Teste de Mann-Whitney apontou diferenças significativas entre as avaliações dos estímulos A3 ${ }^{1}$ e D1 pelos Grupos Controle e Experimental 1 nas escalas FeioBonito $(\mathrm{U}=74,000 ; p<.05)$, Negativo-Positivo ( $\mathrm{U}=$ 70,500; $p<.05)$ e Pobre-Rico ( $\mathrm{U}=56,500 ; p<.05)$; e entre as avaliações de A3 ${ }^{1}$ e D3 realizadas pelos Grupos Controle e Experimental 2 nos resultados das escalas Áspero-Liso $(\mathrm{U}=52,500 ; p<.05)$, Pesado-Leve $(\mathrm{U}=$ 53,000; $p<.05)$, Duro-Macio ( $\mathrm{U}=9,500 ; p<.001)$, Desagradável-Agradável ( $\mathrm{U}=30,500 ; p<.05)$, PassivoAtivo $(\mathrm{U}=54,500 ; p<.05)$ e Pobre-Rico $(\mathrm{U}=31,500 ; p<$ $.001)$.

\section{DISCUSSÃO}

Nesse estudo foi investigada a formação e reorganização de classes de equivalência formadas por estímulos com história pré-experimental, relacionados a profissões, gêneros e adjetivos, e com estímulos sem história pré-experimental (símbolos abstratos), e a ocorrência de transferência de função através da aplicação do Diferencial Semântico. Os resultados obtidos serão analisados e comparados com a literatura que investiga a formação e a reorganização de classes e a transferência de função.

\section{Formação e Reorganização de Classes}

Foi verificado que os participantes dos grupos experimentais atingiram o critério de formação das três classes de equivalência. Na Etapa 2, o Grupo Experimental 1, mesmo com a repetição dos testes, atingiu o critério de reorganização de classes apenas no teste de simetria (Tabela 2). Tais resultados são similares aos de outros estudos que verificaram relações de simetria coerentes com o treino de reversão, mas sem a emergência das relações de transitividade e equivalência (Garrotti \& de Rose, 2007- Experimento 1; Pilgrim \& Galizio, 1990, 1995; Portela, 2014). Garrotti e de Rose (2007) argumentam que tais desempenhos se devem, provavelmente, ao fato de que as relações de simetria envolvem dois conjuntos de estímulos, enquanto que a transitividade e a equivalência compreendem três ou mais conjuntos.

A utilização de estímulos com significado social (profissões e adjetivos), também pode ser considerada como uma variável que afetou a emergência de novas relações incoerentes com a história pré-experimental dos participantes, após os treinos de reversão. Nos testes de reorganização, os participantes do Grupo Experimental 1 deveriam relacionar estímulos de classes diferentes (profissões relacionadas aos gêneros feminino e masculino). Os escores inferiores ao critério (91\% de acerto) nos testes de transitividade e equivalência, realizados após os treinos de reversão, sugerem controle por características do procedimento que diferem das contingências programadas. Adicionalmente, tais desempenhos mostram reorganização parcial das classes e podem indicar efeito da história prévia de aprendizagem, como também foi verificado em outros estudos (Carvalho \& de Rose, 2014; Moxon et al., 1993; Portela, 2014; Watt et al., 1991).

Apesar da utilização do procedimento de DMTS, da realização de treinos de reversão e da inclusão de tentativas de treino previamente aos testes, as classes não foram reorganizadas, o que diverge dos resultados obtidos em outros estudos (Mizael et al., 2016; Ribeiro et al., 2016; Silveira \& de Rose, 2015). Entretanto, os desempenhos nos testes de reorganização foram mais precisos, quando comparados com os resultados de Portela (2014), que utilizou tarefas de pareamento simultâneo e classes com figuras de silhuetas de diferentes biotipos. Com a utilização do DMTS no presente estudo, foi verificada a emergência de relações de simetria para todos os participantes do Grupo Experimental 1 e escores de $83 \%$ nos testes de transitividade e equivalência para seis dos oito participantes, os quais eram inferiores ao critério de reorganização das classes (91\% de acertos). No entanto, Portela (2014) verificou a emergência de simetria para quatro dos seis participantes e escores que variavam de 50 $75 \%$ de acerto nos testes de transitividade e equivalência.

Diferentemente das investigações que demonstraram reorganização de classes, no presente estudo, assim como no estudo de Portela (2014), foram realizados treinos de reversão de duas discriminações condicionais (C1D3, C2D2, C3D1e E1D3, E2D2, E3D1). No treino de reversão, o estímulo D3, originalmente da classe das profissões masculinas, foi relacionado com $\mathrm{C} 1$ (da classe de profissões femininas) e com os adjetivos E1 (mais frequentemente relacionados com uma profissão feminina pelo Grupo Controle). Para o estímulo D1 as relações foram invertidas e não houve mudança para os estímulos da classe relacionada com ambos os gêneros. É possível que o treino EDr, realizado após o treino CDr, tenha distorcido tais discriminações condicionais, mais recentemente treinadas, e afetado o desempenho nos testes de reorganização. Os participantes dos grupos experimentais não foram expostos a tarefa de classificação dos adjetivos em relação a profissões e gêneros antes do estudo. Adicionalmente, tais estímulos não faziam parte das classes de equivalência formadas na Etapa 1.

\section{Transferência de Função}

Quando comparado com o Grupo Controle, as avaliações no Diferencial Semântico dos estímulos A e D foram parcialmente coerentes com as classes de equivalência (Grupo Experimental 2) e com os treinos de reversão e testes de reorganização de classes (Grupo Experimental 1).

Considerando os treinos de reversão (CDr e EDr), para que fosse verificada a transferência de função, as avaliações de D3 (Grupo Experimental 1) deveriam ser similares, em direção e valores no Diferencial Semântico, às avaliações de A1 do Grupo Controle, e as avaliações de $\mathrm{D} 1$ deveriam ser similares as de $\mathrm{A} 3$, pois foram treinadas as relações condicionais C1D3, E1D3, C3D1 e E3D1, 
respectivamente. Para os participantes do Grupo Experimental 1, as avaliações do estímulo D3 foram mais positivas do que as avaliações dos participantes do Grupo Controle para a profissão feminina, que foram mais negativas (A1 ${ }^{1}$ - Empregadora Doméstica); as avaliações do estímulo D1 tenderam a ser mais positivas do que as avaliações da profissão masculina (A3 ${ }^{1}$ - Mecânico) do Grupo Controle (ver Figura 2). Entretanto, as diferenças não foram significativas para todas as escalas, o que é coerente com os resultados do teste de reorganização (Tabela 1) que mostraram que após o treino de reversão ocorreu reorganização parcial das classes.

$\mathrm{Na}$ maioria das escalas, o Grupo Experimental 2 avaliou o estímulo D1 de maneira similar às avaliações da profissão feminina (A1 ${ }^{1}$ - Empregadora Doméstica) do Grupo Controle, o que está de acordo com as classes formadas na Etapa 1. Por outro lado, as avaliações do estímulo D3 foram mais positivas e/ou na direção oposta das avaliações da profissão masculina (A3 ${ }^{1}$ - Mecânico). Tal resultado não é coerente com as contingências as quais o Grupo Experimental 2 foi exposto, que não incluíam o treino de reversão. Considerando que na Etapa 1 foi verificada a formação de três classes de equivalência para todos os participantes, era mais provável que fosse verificada a transferência de função entre os membros da classe relacionada com a profissão masculina (A3, B3, C3 e D3), ou seja, D3 deveria ter sido avaliado no Diferencial Semântico de maneira similar a Mecânico (A3 $\left.{ }^{1}\right)$.

É possível que variáveis relacionadas ao contexto cultural tenham afetado as avaliações dos participantes. De acordo com a visão da sociologia funcionalista acerca das profissões, o estímulo A3 ${ }^{1}$ (Mecânico), pode ter sido avaliado de maneira negativa pelo Grupo Controle por tratar-se de uma profissão considerada de baixa qualificação, em comparação com profissões que demandam formação a nível de graduação. Em algumas culturas, empregos considerados menos qualificados podem não ter seu valor reconhecido socialmente (Dubar, 2012). Assim, história de aprendizagem similar pode ter afetado as avaliações nas escalas do Diferencial Semântico.

Adicionalmente, a história anterior de aprendizagem dos participantes dos grupos experimentais em relação a gêneros, profissões, e adjetivos não foi previamente avaliada, pois os estímulos foram definidos a partir de um levantamento com estudantes que não foram expostos as tarefas experimentais. Essa diferença em relação ao estudo de Mizael et al. (2016), que treinou relações condicionais entre faces de afrodescendentes e símbolo positivo contrárias ao viés racial negativo previamente identificado, pode estar relacionada com a falha em demonstrar a reorganização das classes no presente estudo.

Outro aspecto relevante envolve as características das escalas do Diferencial Semântico e a modalidade dos estímulos com significado social. Quando são utilizados estímulos abstratos e faces (e.g., alegres, neutras e raivosas), as escalas do Diferencial Semântico podem ser classificadas de acordo com o Fator 1 (Avaliação) e o Fator 2 (Potência). O Fator 1 está relacionado com características físicas e afetivas (escalas Triste-Alegre, Tenso-Relaxado, Áspero-Liso, Feio-Bonito, Pesado-Leve, Negativo-Positivo, Duro-Macio, Mau-Bom e Desagradável-Agradável) e o Fator 2 com qualidades de atividade e potência (escalas Lento-Rápido, Passivo-Ativo, Pobre-Rico e Submisso-Dominante). As escalas do Fator 1 mostraram alto índice de correlação entre si e valores com maior variação nas medianas. Entretanto, nas escalas do Fator 2 o índice de correlação é menor e os valores apresentados nas medianas são mais neutros e com menores variações (Almeida \& de Rose, 2015).

No presente estudo, que utilizou estímulos com significado social relacionados a profissões e gênero, foram observadas avaliações com escores altos e com maiores variações nas escalas do Fator 2, que são compostas por palavras qualificadores de atividade e potência. Desta forma, as escalas Lento-Rápido, PassivoAtivo, Pobre-Rico e Submisso-Dominante (Fator 2) provavelmente controlaram o responder (avaliações diferentes de zero) dos participantes, pois eram mais similares a relações previamente aprendidas entre profissões e gênero no contexto social do que as faces.

Outra variável relevante para a formação de classes e a transferência de função é a estrutura do treino (Arntzen, 2012; Bortoloti \& de Rose, 2009; Saunders \& Green, 1999). No estudo de Almeida e de Rose (2015), o treino de reversão $\mathrm{CDr}$ e os testes de reorganização foram realizados após os treinos das relações condicionais $A B$, $\mathrm{AC}, \mathrm{CD}$ e a verificação da formação de classes de equivalência, sendo que os testes foram apenas das relações BD e DB. Na Etapa 1 do presente estudo, foram treinadas as relações $\mathrm{AC}, \mathrm{BC}$ e $\mathrm{CD}$ e testadas relações simétricas ( $\mathrm{CA}, \mathrm{CB}$ e $\mathrm{DC})$, de transitividade $(\mathrm{AB}, \mathrm{BD}$ e $\mathrm{AD})$ e equivalência (BA, DB e DA). Na Etapa 2 foram revertidas duas relações (CDr e EDr) e testadas todas as possíveis relações entre os estímulos. Considerando, que A era o conjunto das profissões, optou-se por um arranjo de treino em que o conjunto $\mathrm{C}$ (formas abstratas) fosse o elo comum e que apenas uma das relações condicionais incluísse as profissões. Diferentemente, no estudo de Almeida e de Rose (2015), as faces (A) foram relacionadas com os estímulos B e C. É possível que a estrutura do treino, que envolvia diferentes aprendizagens de discriminações simples e condicionais entre os vários treinos e a quantidade de testes realizados, possa estar relacionada com a diferença entre os dois estudos em relação a reorganização das classes e as avalições dos estímulos com o Diferencial Semântico.

\section{CONSIDERAÇÕES FINAIS}

Considerando que o gênero é determinado pela cultura e mantido por meio de contingências sociais em uma comunidade verbal (Ruiz, 2003), práticas culturais e costumes, tais como a associação feita entre o masculino com contextos públicos e do trabalho e o feminino com contextos privados, cuidados com o lar e da família, envolvem relações condicionais que são aprendidas e podem ser modificadas. Os procedimentos utilizados no presente estudo permitiram investigar processos envolvidos na formação e modificação de classes com 
estímulos relacionados a profissões e gêneros. Nesse sentido, pode-se considerar a generalidade do procedimento para questões sociais relacionadas a estereótipos de gênero. A investigação de tais questões é relevante e viável a partir da perspectiva da Análise do Comportamento, e pode contribuir para a identificação de variáveis envolvidas na formação e modificação dessas relações não igualitárias e para o planejamento de contingências de intervenção.

Entretanto, a reorganização parcial das classes de equivalência, ou a emergência de parte das relações condicionais, e a transferência de função de apenas algumas escalas indicam a necessidade de aprimoramento de controles metodológicos em relação a verificação da história pré-experimental com os estímulos utilizados e a quantidade de relações revertidas. No caso da transferência de função, os resultados sugerem análises adicionais em relação as diferentes escalas do Diferencial Semântico quanto aos fatores 1 e 2, quando são utilizados estímulos diferentes de faces.

Por fim, pesquisas sobre reorganização de classes permitem investigar contingências de reforçamento que modificam relações entre estímulos que são a base de classes previamente estabelecidas, e produzam reorganização de tais classes ou até mesmo responder sob controle de classes diferentes em contextos distintos. Tais estudos possuem implicações teóricas e práticas, principalmente no contexto educacional e clínico, e contribuem para o desenvolvimento de intervenções que favoreçam a modificação de comportamentos complexos (Regra, 2010; Sidman, 1994).

\section{DECLARAÇÃO DE CONFLITO DE INTERESSES}

As autoras declaram que não há qualquer conflito de interesses relativos à publicação deste artigo.

\section{CONTRIBUIÇÃO DE CADA AUTORA}

Certificamos que ambas as autoras participaram suficientemente do trabalho para tornar pública sua responsabilidade pelo conteúdo. A contribuição de cada autora pode ser atribuída como se segue: A. P. Rosendo e R. M. Melo contribuíram para a concepção do artigo; A. P. Rosendo e R. M. Melo foram responsáveis pela formulação do design metodológico; A. P. Rosendo fez a coleta de dados; e A. P. Rosendo e R. M. Melo foram responsáveis pela redação final.

\section{DIREITOS AUTORAIS}

Este é um artigo aberto e pode ser reproduzido livremente, distribuído, transmitido ou modificado, por qualquer pessoa desde que usado sem fins comerciais. O trabalho é disponibilizado sob a licença Creative Commons 4.0 BY-NC.

\section{(cc) EY-NC}

\section{REFERÊNCIAS}

Albuquerque, A. R., \& Melo, R. M. (2005). Equivalência de estímulos: conceito, implicações e possibilidades de aplicação. In J. Abreu-Rodrigues \& M. R. Ribeiro (Orgs.), Análise do Comportamento: Pesquisa, teoria e aplicação (pp. 245-264). Porto Alegre: Artmed.

Arntzen, E. (2012). Training and testing parameters in formation of stimulus equivalence: Metodological issues. European Journal of Behavior Analysis, 13, 123-135.

Almeida, J. H., Bortoloti, R., Ferreira, P. R. S., Schielini, P. W., \& de Rose, J. C. (2014). Análise da validade e precisão de instrumento de diferencial semântico. Psicologia: Reflexão e Crítica, 27, 272-281.

Almeida, J. H., \& de Rose, J. C. (2015). Changing the meaningfulness of abstract stimuli by the reorganization of equivalence classes: Effects of delayed matching. The Psychological Record, 65, 451461.

Bortoloti, R., \& de Rose, J. C. (2007). Medida do grau de relacionamento entre estímulos equivalentes. Psicologia: Reflexão e Crítica, 20, 252258.

Bortoloti, R., \& de Rose, J. C. (2009). Assessment of the relatedness of equivalent stimuli through a semantic differential. The Psychological Record, 59, 563-590.

Bortoloti, R., \& de Rose, J. C. (2012). Equivalent stimuli are more strongly related after training with delayed matching than after simultaneous matching: A study using the implicit relational assessment procedure (IRAP). The Psychological Record, 62, 41-54.

Cardoso, A. L. (2013). Efeito da quantidade de treino na reorganização das classes de equivalência em crianças. (Dissertação de mestrado não publicada). Universidade de Brasília, Brasília.

Carvalho, M. P., \& de Rose, J. C. (2014). Understanding racial attitudes through the stimulus equivalence paradigm. The Psychological Record, 64, 527-536.

de Rose, J. C., \& Bortoloti, R. (2007). A equivalência de estímulos como modelo do significado. Acta Comportamentalia, 15, 88-102.

Dixon, M. R., Rehfeldt, R. A., Zlomke, K. R., \& Robinson, A. (2006). Exploring the development and dismantling of equivalence classes involving terrorist stimuli. The Psychological Record, 56, 83-103.

Dougher, M. J., \& Markham, M. R. (1994). Stimulus equivalence, functional equivalence and the transfer of function. In S. C. Hayes, L. J. Hayes, M. Sato \& K. Ono (Orgs.), Behavior analysis of language and cognition (pp. 71-90). Reno, NV: Context Press.

Dubar, C. (2012). A construção de si pela atividade de trabalho: A socialização profissional. Cadernos de Pesquisa, 42, 351-367.

Garotti, M., \& de Rose, J. C. (2007). Reorganization of equivalence classes: Evidence for contextual control by baseline reviews before probes. The Psychological Record, 57, 87-102.

Hanna, E. S., Batitucci, L. A. V., \& Batitucci, J. S. L. (2014). Software Contingência Programada: Utilidade 
e funcionalidades. Revista Brasileira de Análise do Comportamento, 10, 97-104.

Haydu, V. B., Camargo, J., \& Bayer, H. (2015). Effects of pre-experimental history on the formation of stimulus equivalence classes: A study with supporters of Brazilian soccer clubs. Psychology \& Neuroscience, 8 , 385-396.

Mizael, T. M., Almeida, J. H., Silveira, C. C., de Rose, J. C. (2016). Changing racial bias by transfer of functions in equivalence classes. The Psychological Record, 66, 451-462.

Moxon, P. D., Keenan, M., \& Hine, L. (1993). Genderrole stereotyping and stimulus equivalence. The Psychological Record, 43, 381-394.

Nalini, L. E. G. (2002). Determinação empírica da nomeabilidade de estímulos: implicações para o estudo da relação de nomeação. (Tese de doutorado não publicada). Universidade de Brasília, Brasília.

Pilgrim, C., \& Galizio, M. (1990). Relations between baseline contingencies and equivalence probe performances. Journal of the Experimental Analysis of Behavior, 54, 213-224.

Pilgrim, C., \& Galizio, M. (1995). Reversal of baseline relations and stimulus equivalence: I. Adults. Journal of the Experimental Analysis of Behavior, 63, 225-238.

Portela, L. C. D. S. (2014). Transferência de função e reorganização de classes de equivalência relacionadas com diferentes biotipos. (Dissertação de mestrado não publicada). Universidade de Brasília, Brasília.

Regra, J. A. G. (2010). Formação de classes de respostas, resistência à mudança e terapia comportamental infantil. Perspectivas em Análise do Comportamento, 1, 93-103.

Ribeiro, G. W., Silveira, M. V., Mackay, H. A., \& de Rose, J. C. (2016). The effect of conditional discrimination reversals with SMTS and DMTS on reorganization of equivalence classes. The Psychological Record, 66, 589-597.

Ruiz, M. R. (2003). Inconspicuous sources of behavioral control: The case of gendered practices. The Behavior Analyst Today, 4, 12-16.

Saunders, R. R., \& Green, G. (1999). A discrimination analysis of training-structure effects on stimulus equivalence outcomes. Journal of the Experimental Analysis of Behavior, 72, 117-137.

Sidman, M. (1994). Equivalence relations and behavior: A research history. Boston, MA: Authors Cooperative, Inc., Publishers.

Sidman, M., \& Tailby, W. (1982). Conditional discrimination versus matching to sample: An expansion of the testing paradigm. Journal of the Experimental Analysis of Behavior, 37, 5-22.

Watt, A., Keenan, M., Barnes, D., \& Cairns, E. (1991). Social categorization and stimulus equivalence. The Psychological Record, 41, 33-50.

Submetido em: 08/05/2018 Aceito em: 09/11/2018 